\title{
Closed form solutions of Lyapunov equations using the vech and veck operators
}

\author{
NICHOLAS ASSIMAKIS \\ General Department \\ National and Kapodistrian University of Athens \\ Psachna Evias \\ GREECE \\ MARIA ADAM \\ Department of Computer Science and Biomedical Informatics \\ University of Thessaly \\ Lamia \\ GREECE
}

\begin{abstract}
New closed forms are presented of the solutions of the continuous and discrete Lyapunov equations using the vech and veck operators. The proposed solutions are faster than the classical solutions derived using the vec operator. The solutions via veck operator are faster than the solutions via vech operator.
\end{abstract}

Key-Words: - continuous Lyapunov equation, discrete Lyapunov equation, vec, vech, veck operator

Received: January 12, 2021. Revised: May 6, 2021. Accepted: May 30, 2021. Published: June 10, 2021.

\section{Introduction}

Matrix equations [1]-[3] play a fundamental role in many tasks in control theory. Lyapunov equations play a very important role in stability theory of continuous systems [4] and discrete systems [4], [5].

The continuous Lyapunov equation is associated with continuous state space systems.

\section{Continuous Lyapunov equation}

Consider the $\mathrm{n} \times \mathrm{n}$ real square matrices $\mathrm{F}, \mathrm{Q}$, where $Q$ is symmetric and nonnegative definite. The continuous Lyapunov equation is

$\mathrm{F} \cdot \mathrm{X}+\mathrm{X} \cdot \mathrm{F}^{\mathrm{T}}=-\mathrm{Q}$

where the solution $\mathrm{X}$ is an $\mathrm{n} \times \mathrm{n}$ real square symmetric nonnegative definite matrix. $\mathrm{F}^{\mathrm{T}}$ denotes the transpose of $\mathrm{F}$.

Existence and uniqueness of solution.

Let $\lambda_{\mathrm{i}}, \mathrm{i}=1 \ldots \mathrm{n}$ be the eigenvalues of $\mathrm{F}$. The continuous Lyapunov equation (1.1) has a unique symmetric nonnegative definite solution $X$ if and only if $\lambda_{i} \neq-\lambda_{j}$ for all $i, j=1, \ldots, n,[2]$.

The discrete Lyapunov equation is associated with discrete state space systems.

\section{Discrete Lyapunov equation}

Consider the $\mathrm{n} \times \mathrm{n}$ real square matrices $\mathrm{F}, \mathrm{Q}$, where $Q$ is symmetric and nonnegative definite. The discrete Lyapunov equation is

$\mathrm{X}=\mathrm{Q}+\mathrm{F} \cdot \mathrm{X} \cdot \mathrm{F}^{\mathrm{T}}$ where the solution $\mathrm{X}$ is an $\mathrm{n} \times \mathrm{n}$ real square symmetric nonnegative definite matrix.

\section{Existence and uniqueness of solution.}

Let $\lambda_{\mathrm{i}}, \mathrm{i}=1 \ldots \mathrm{n}$ be the eigenvalues of $\mathrm{F}$. The discrete Lyapunov equation (1.2) has an unique symmetric nonnegative definite solution $\mathrm{X}$ if and only if $\left|\lambda_{\mathrm{i}}\right|<1$ for all $\mathrm{i}=1, \ldots, \mathrm{n}$.

In the literature [6]-[9] there exist iterative as well as algebraic solutions of the continuous Lyapunov equation (for instance Arnoldi method, Smith's algorithm, Hessenberg-Shur method).

In the literature [5] there exist iterative as well as algebraic solutions of the discrete Lyapunov equation (for instance Chandrasekhar type algorithms, doubling algorithm, Vaughan's algebraic non-recursive solution).

Both continuous and discrete Lyapunov equations are equivalent to linear systems of equations. Thus solutions via the vec operator are derived. Classical closed forms solutions using the vec operator are briefly presented in section II.

In this paper, new closed forms are proposed for the solutions of the continuous and discrete Lyapunov equations using the operator vech and the operator veck, in sections III and IV, respectively. The computational requirements of the proposed method are determined in section V. It is shown that the proposed solutions derived using the vech and 
veck operators are faster than the classical solutions derived using the vec operator. Finally, Section VI summarizes the conclusions.

The novelty of this paper concerns a) the analytic determination of the computational requirements of the algorithms that use the vec, vech and veck operators, with respect to the matrices dimension $n$, b) the derivation of the speedup form the classical solution via vec operator to the proposed solutions via vech and veck operators.

\section{Solutions Using vec Operator}

Lyapunov equations involve matrices. Then we are able to use the vec operator, which stacks columns of a matrix one under another in a single column.

\section{Continuous Lyapunov equation}

$\mathrm{F} \cdot \mathrm{X}+\mathrm{X} \cdot \mathrm{F}^{\mathrm{T}}=-\mathrm{Q}$

$$
\begin{aligned}
\Rightarrow \operatorname{vec}(-Q) & =\operatorname{vec}\left(F \cdot X+X \cdot F^{T}\right) \\
& =\operatorname{vec}\left(F \cdot X \cdot I_{n}\right)+\operatorname{vec}\left(I_{n} \cdot X \cdot F^{T}\right) \\
& =\left(I_{n} \otimes F\right) \cdot \operatorname{vec}(X)+\left(F \otimes I_{n}\right) \cdot \operatorname{vec}(X)
\end{aligned}
$$

where $I_{n}$ denotes the he $n \times n$ identity matrix, $\otimes$ is the Kronecker product and the following properties [10], [11] were used:

$$
\begin{aligned}
& \operatorname{vec}(A+B)=\operatorname{vec}(A)+\operatorname{vec}(B) \\
& \operatorname{vec}(A \cdot B \cdot C)=\left(C^{T} \otimes A\right) \cdot \operatorname{vec}(B) \\
& \quad \text { Then, defining } \\
& C=I_{n} \otimes F+F \otimes I_{n} \\
& \text { we get: } \\
& C \cdot \operatorname{vec}(X)=-\operatorname{vec}(Q)
\end{aligned}
$$

Then, if the conditions for the existence of a unique solution of the continuous Lyapunov equation are satisfied, then the matrix $\mathrm{C}$ is nonsingular and we get:

$\operatorname{vec}(\mathrm{X})=-\mathrm{C}^{-1} \cdot \operatorname{vec}(\mathrm{Q})$

The construction of the solution $\mathrm{X}$ from $\operatorname{vec}(\mathrm{X})$ is trivial.

\section{Discrete Lyapunov equation}

$$
\begin{aligned}
& X=Q+F \cdot X \cdot F^{T} \\
& \begin{aligned}
\Rightarrow \operatorname{vec}(X) & =\operatorname{vec}\left(Q+F \cdot X \cdot F^{T}\right) \\
& =\operatorname{vec}(Q)+\operatorname{vec}\left(F \cdot X \cdot F^{T}\right) \\
& =\operatorname{vec}(Q)+(F \otimes F) \cdot \operatorname{vec}(X)
\end{aligned}
\end{aligned}
$$

Then, defining

$\mathrm{C}=\mathrm{I}_{\mathrm{n}} \otimes \mathrm{I}_{\mathrm{n}}-\mathrm{F} \otimes \mathrm{F}$

we get:

$$
\mathrm{C} \cdot \operatorname{vec}(\mathrm{X})=\operatorname{vec}(\mathrm{Q})
$$

Then, if the conditions for the existence of a unique solution of the discrete Lyapunov equation are satisfied, then the matrix $C$ is nonsingular and we get:

$$
\operatorname{vec}(\mathrm{X})=\mathrm{C}^{-1} \cdot \operatorname{vec}(\mathrm{Q})
$$

The construction of the solution $\mathrm{X}$ from $\operatorname{vec}(\mathrm{X})$ is trivial.

\section{Solutions Using vech Operator}

Lyapunov equations involve symmetric matrices. Then we are able to use the vech operator, which stacks columns of a square matrix one under another in a single column, starting each column at its diagonal element.

The relation between the vec operator and the vech operator is described using the duplication matrix and the elimination matrix. For a symmetric matrix $S$, we also use the $n^{2} \times \frac{n(n+1)}{2}$ dimensional duplication matrix $D_{n}$ and $\frac{n(n+1)}{2} \times n^{2}$ dimensional elimination matrix $L_{n}$ :

$\mathrm{D}_{\mathrm{n}} \cdot \operatorname{vech}(\mathrm{S})=\operatorname{vec}(\mathrm{S})$

$\mathrm{L}_{\mathrm{n}} \cdot \operatorname{vec}(\mathrm{S})=\operatorname{vech}(\mathrm{S})$

The knowledge of the duplication matrix and the elimination matrix allows the derivation of the Lyapunov equations via vech operator.

\section{Continuous Lyapunov equation}

Multiplying (2.2) by $\mathrm{L}_{n}$ using (3.1) and (3.2) we get:

$$
\begin{aligned}
& \mathrm{C} \cdot \operatorname{vec}(X)=-\operatorname{vec}(\mathrm{Q}) \\
& \Rightarrow \mathrm{L}_{\mathrm{n}} \cdot \mathrm{C} \cdot \operatorname{vec}(\mathrm{X})=-\mathrm{L}_{\mathrm{n}} \cdot \operatorname{vec}(\mathrm{Q})=-\operatorname{vech}(\mathrm{Q}) \\
& \Rightarrow \mathrm{L}_{\mathrm{n}} \cdot \mathrm{C} \cdot \mathrm{D}_{\mathrm{n}} \cdot \operatorname{vech}(\mathrm{X})=-\operatorname{vech}(\mathrm{Q}) \\
& \quad \text { Then, defining } \\
& \mathrm{E}=\mathrm{L}_{\mathrm{n}} \cdot \mathrm{C} \cdot \mathrm{D}_{\mathrm{n}} \\
& \text { we get } \\
& \mathrm{E} \cdot \operatorname{vech}(\mathrm{X})=-\operatorname{vech}(\mathrm{Q})
\end{aligned}
$$

From (3.3) the nonsingularity of $\mathrm{C}$ yields the nonsingularity of $E$, [2]. Hence, the equation in (3.4) follows

$\operatorname{vech}(\mathrm{X})=-\mathrm{E}^{-1} \cdot \operatorname{vech}(\mathrm{Q})$

The construction of the solution $\mathrm{X}$ from vech $(\mathrm{X})$ is trivial.

\section{Discrete Lyapunov equation}

Multiplying (2.5) by $\mathrm{L}_{\mathrm{n}}$ using (3.1) and (3.2) we get:

$$
\begin{aligned}
& \mathrm{C} \cdot \operatorname{vec}(X)=\operatorname{vec}(Q) \\
& \quad \Rightarrow L_{n} \cdot C \cdot \operatorname{vec}(X)=L_{n} \cdot \operatorname{vec}(Q)=\operatorname{vech}(Q) \\
& \quad \Rightarrow L_{n} \cdot C \cdot D_{n} \cdot \operatorname{vech}(X)=\operatorname{vech}(Q) \\
& \quad \text { Then, defining } \\
& \text { E }=L_{n} \cdot C \cdot D_{n} \\
& \text { we get } \\
& E \cdot \operatorname{vech}(X)=\operatorname{vech}(Q)
\end{aligned}
$$

From (3.6) the nonsingularity of $\mathrm{C}$ yields the nonsingularity of $\mathrm{E},[2]$. Hence, the equation in (3.7) follows

$\operatorname{vech}(\mathrm{X})=\mathrm{E}^{-1} \cdot \operatorname{vech}(\mathrm{Q})$

The construction of the solution $\mathrm{X}$ from vech(X) is trivial. 


\section{Solutions Using veck Operator}

Lyapunov equations can be written in a form using skew symmetric matrices. Then we are able to use the veck operator [12], [13], which functions like vech operator and removes the zero elements of the main diagonal.

The relation between the vec operator and the veck operator is described using the duplication matrix. For a skew symmetric matrix s, we also use the $\mathrm{n}^{2} \times \frac{\mathrm{n}(\mathrm{n}-1)}{2}$ dimensional duplication matrix $d_{n}$ :

$\mathrm{d}_{\mathrm{n}} \cdot \operatorname{veck}(\mathrm{s})=\operatorname{vec}(\mathrm{s})$

$\frac{1}{2} \cdot d_{\mathrm{n}}^{\mathrm{T}} \cdot \operatorname{vec}(\mathrm{s})=\operatorname{veck}(\mathrm{s})$

The knowledge of the duplication matrix allows the derivation of the Lyapunov equations via veck operator.

Continuous Lyapunov equation

The equivalent formula in (1.1) is written

$\mathrm{F} \cdot \mathrm{S}+\mathrm{S} \cdot \mathrm{F}^{\mathrm{T}}=-\mathrm{R}$

where

$\mathrm{S}=\mathrm{F} \cdot \mathrm{X}-\mathrm{X} \cdot \mathrm{F}^{\mathrm{T}}$

$\mathrm{R}=\mathrm{F} \cdot \mathrm{Q}-\mathrm{Q} \cdot \mathrm{F}^{\mathrm{T}}$

are skew symmetric matrices, [14].

Then, defining

$\mathrm{C}=\mathrm{I}_{\mathrm{n}} \otimes \mathrm{F}+\mathrm{F} \otimes \mathrm{I}_{\mathrm{n}}$

we get:

$\mathrm{C} \cdot \operatorname{vec}(\mathrm{S})=\operatorname{vec}(-\mathrm{R})$

Then defining

$\mathrm{D}=\frac{1}{2} \cdot \mathrm{d}_{\mathrm{n}}^{\mathrm{T}} \cdot \mathrm{C} \cdot \mathrm{d}_{\mathrm{n}}$

we get

$\mathrm{D} \cdot \operatorname{veck}(\mathrm{S})=\operatorname{veck}(-\mathrm{R})$

From (4.8) the nonsingularity of $\mathrm{C}$ yields the nonsingularity of $\mathrm{D},[2]$. Hence, the equation in (4.9) follows

$\operatorname{veck}(\mathrm{S})=\mathrm{D}^{-1} \cdot \operatorname{veck}(-\mathrm{R})$

The construction of the solution $S$ from $\operatorname{veck}(S)$ is trivial.

Finally, the solution of (1.1) is

$\mathrm{X}=\frac{1}{2} \cdot \mathrm{F}^{-1} \cdot(\mathrm{S}-\mathrm{Q})=-\frac{1}{2} \cdot(\mathrm{S}+\mathrm{Q}) \cdot \mathrm{F}^{-\mathrm{T}}$

\section{Discrete Lyapunov equation}

Equation (1.2) is equivalent to

$\mathrm{S}=\mathrm{R}+\mathrm{F} \cdot \mathrm{S} \cdot \mathrm{F}^{\mathrm{T}}$

where

$\mathrm{S}=\mathrm{F} \cdot \mathrm{X}-\mathrm{X} \cdot \mathrm{F}^{\mathrm{T}}$

$\mathrm{R}=\mathrm{F} \cdot \mathrm{Q}-\mathrm{Q} \cdot \mathrm{F}^{\mathrm{T}}$

are skew symmetric matrices.

Then, defining

$\mathrm{C}=\mathrm{I}_{\mathrm{n}} \otimes \mathrm{I}_{\mathrm{n}}-\mathrm{F} \otimes \mathrm{F}$

we get:

$\mathrm{C} \cdot \operatorname{vec}(\mathrm{S})=\operatorname{vec}(\mathrm{R})$

$\mathrm{D}=\frac{1}{2} \cdot \mathrm{d}_{\mathrm{n}}^{\mathrm{T}} \cdot \mathrm{C} \cdot \mathrm{d}_{\mathrm{n}}$
$\mathrm{D} \cdot \operatorname{veck}(\mathrm{S})=\operatorname{veck}(\mathrm{R})$

From (4.17) the nonsingularity of $\mathrm{C}$ yields the nonsingularity of $\mathrm{D},[2]$. Hence, the equation in (4.18) follows

$\operatorname{veck}(\mathrm{S})=\mathrm{D}^{-1} \cdot \operatorname{veck}(\mathrm{R})$

The construction of the solution $S$ from $\operatorname{veck}(\mathrm{S})$ is trivial.

Finally, the solution of (1.2) is

$\mathrm{X}=(\mathrm{I}-\mathrm{F} \cdot \mathrm{F})^{-1} \cdot(\mathrm{Q}-\mathrm{F} \cdot \mathrm{S})$

$=\left(\mathrm{Q}+\mathrm{S} \cdot \mathrm{F}^{\mathrm{T}}\right) \cdot\left(\mathrm{I}-\mathrm{F}^{\mathrm{T}} \cdot \mathrm{F}^{\mathrm{T}}\right)^{-1}$

All continuous and discrete Lyapunov equations solutions via vec, vech and veck operators are summarized in Table I.

TABLE I. LYAPUNOV EQUATIONS SOLUTIONS VIA VEC, VECH

\begin{tabular}{|c|c|c|}
\multicolumn{2}{|c|}{ AND VECK OPERATORS } \\
\hline & $\begin{array}{c}\text { Continuous } \\
\text { Lyapunov } \\
\text { Equation }\end{array}$ & $\begin{array}{c}\text { Discrete } \\
\text { Lyapunov } \\
\text { Equation }\end{array}$ \\
& $F \cdot X+X \cdot F^{T}=-Q$ & $X=Q+F \cdot X \cdot F^{T}$ \\
\hline $\begin{array}{c}\text { Use } \\
\text { of }\end{array}$ & $C=I_{n} \otimes F+F \otimes I_{n}$ & $C=I_{n} \otimes I_{n}-F \otimes F$ \\
vec & vec $(X)=-C^{-1} \cdot \operatorname{vec}(Q)$ & $\operatorname{vec}(X)=C^{-1} \cdot \operatorname{vec}(Q)$ \\
\hline $\begin{array}{c}\text { Use } \\
\text { of }\end{array}$ & $C=I_{n} \otimes F+F \otimes I_{n}$ & $C=I_{n} \otimes I_{n}-F \otimes F$ \\
vech & $E=L_{n} \cdot C \cdot D_{n}$ & $E=L_{n} \cdot C \cdot D_{n}$ \\
\hline & vech $(X)=-E^{-1} \cdot \operatorname{vech}(Q)$ & vech $(X)=E^{-1} \cdot \operatorname{vech}(Q)$ \\
& $C=I_{n} \otimes F+F \otimes I_{n}$ & $C=I_{n} \otimes I_{n}-F \otimes F$ \\
Use & $R=F \cdot Q-Q \cdot F^{T}$ & $R=F \cdot Q-Q \cdot F^{T}$ \\
of & $D=\frac{1}{2} \cdot d_{n}^{T} \cdot C \cdot d_{n}$ & $D=\frac{1}{2} \cdot d_{n}^{T} \cdot C \cdot d_{n}$ \\
veck & veck $(S)=D^{-1} \cdot \operatorname{veck}(-R)$ & veck $(S)=D^{-1} \cdot \operatorname{veck}(R)$ \\
& $X=\frac{1}{2} \cdot F^{-1} \cdot(S-Q)$ & $X=(I-F \cdot F)^{-1} \cdot(Q-F \cdot S)$ \\
& $=-\frac{1}{2} \cdot(S+Q) \cdot F^{-T}$ & $=\left(Q+S \cdot F^{T}\right) \cdot\left(I-F^{T} \cdot F^{T}\right)^{-1}$ \\
\hline
\end{tabular}

\section{Example 1. Continuous Lyapunov equation.}

A numerical example for an ill-conditioned continuous Lyapunov equation is taken from [15] with $\mathrm{n}=3$. Consider the continuous Lyapunov equation with

$\mathrm{F}=\left[\begin{array}{ccc}1 & 0 & 0 \\ 1 & 0.0001 & 0 \\ 1 & 1 & 1\end{array}\right]$

$Q=-\left[\begin{array}{ccc}2 & 2.0001 & 4 \\ 2.0001 & 2.0002 & 4.0001 \\ 4 & 4.0001 & 6\end{array}\right]$

Then

$\mathrm{n}^{2}=9$ and $\mathrm{C}$ is a $9 \times 9$ dimensional matrix.

$\mathrm{m}=6$ and $\mathrm{E}$ is a $6 \times 6$ dimensional matrix.

$\mathrm{k}=3$ and $\mathrm{D}$ is a $3 \times 3$ dimensional matrix.

The solution of the continuous Lyapunov equation is

$X=\left[\begin{array}{lll}1 & 1 & 1 \\ 1 & 1 & 1 \\ 1 & 1 & 1\end{array}\right]$

\section{Example 2. Discrete Lyapunov equation.}

A numerical example for a linearized model of an F-8 aircraft is taken from [15] with $\mathrm{n}=4$. Consider the discrete Lyapunov equation with 
$F=10^{-3} \cdot\left[\begin{array}{cccc}998.51 & -8.044 & -0.10886 & -0.018697 \\ 0.15659 & 1000 & -0.76232 & 3.2272 \\ -213.94 & 0.88081 & 897.21 & 92.826 \\ 110.17 & -0.37821 & -445.56 & 929.68\end{array}\right]$

$\mathrm{Q}=0.1 \cdot \mathrm{I}_{4}$

Then

$\mathrm{n}^{2}=16$ and $\mathrm{C}$ is a $16 \times 16$ dimensional matrix.

$\mathrm{m}=10$ and $\mathrm{E}$ is a $10 \times 10$ dimensional matrix.

$\mathrm{k}=6$ and $\mathrm{D}$ is a $6 \times 6$ dimensional matrix.

The solution of the discrete Lyapunov equation is

X

$=\left[\begin{array}{cccc}76.6687 & -7.9849 & -7.8654 & 168.2162 \\ -7.9849 & 71.1428 & 3.1292 & -15.0098 \\ -7.8654 & 3.1292 & 1.7374 & -17.0180 \\ 168.2162 & -15.0098 & -17.0180 & 373.7570\end{array}\right]$

\section{Computational Requirements}

In order to investigate possible computational advantages of the proposed solutions versus the classical solutions, we are going to compare them. Thus, we compare the algorithms with respect to their computational burdens.

From Table I, it is clear that the Lyapunov equations solutions via vec, vech and veck operators, involve matrix manipulation operations: matrix addition, multiplication and inversion.

Scalar operations are involved in matrix manipulation operations, which are needed for the implementation of the solutions. Table II summarizes the calculation burden of needed matrix operations for the general multidimensional case, where $\geq 2, m \geq 2, k \geq 2$. The details are given in [16].

TABLE II. CALCUlation BURDEN OF MATRIX Operations

\begin{tabular}{|c|c|c|}
\hline Matrix Operation & Matrix Dimensions & Calculation Burden \\
\hline$s \cdot A, s \neq 0, s \neq 1$ & $n \times n$ & $n^{2}$ \\
\hline $0 \cdot A$ & $n \times n$ & 0 \\
\hline $1 \cdot A$ & $n \times n$ & 0 \\
\hline$C=I_{n}+A$ & $(n \times n)+(n \times n)$ & $n$ \\
\hline$C=A+B$ & $(n \times n)+(n \times n)$ & $n^{2}$ \\
\hline$C=A-A^{T}$ & $(n \times n)+(n \times n)$ & $\frac{1}{2} n(n-1)$ \\
\hline$C=A \cdot B$ & $(n \times m) \cdot(m \times k)$ & $2 n m k-n k$ \\
\hline$C^{-1}$ & $n \times n$ & $\frac{1}{6}\left(16 n^{3}-3 n^{2}-n\right)$ \\
\hline
\end{tabular}

Note that the classical solutions that use vec, require the computation of the inverse of a matrix dimensions $\mathrm{N} \times \mathrm{N}$ where $\mathrm{N}=\mathrm{n}^{2}$, while the proposed solutions that use vech, require the computation of the inverse of a matrix of dimensions $\mathrm{m} \times \mathrm{m}$ where $\mathrm{m}=\frac{\mathrm{n}(\mathrm{n}+1)}{2}$ and the proposed solutions that use veck, require the computation of the inverse of a matrix of dimensions $\mathrm{k} \times \mathrm{k}$ where $\mathrm{k}=\frac{\mathrm{n}(\mathrm{n}-1)}{2}$.

Note that no scalar operations are required in order construction the solution $\mathrm{X}$ from $\operatorname{vec}(\mathrm{X})$ or $\operatorname{vech}(\mathrm{X})$.

The calculation burdens of the classical and the proposed Lyapunov equations solutions are analytically calculated at the Appendix and summarized in Table III.

TABLE III. CALCUlation BuRdens OF LyapunOv EQuATIONS SOLUTIONS

\begin{tabular}{|c|c|}
\hline \multicolumn{2}{|c|}{ Classical solutions - Use of vec } \\
\hline Matrix Equation & $\frac{16 n^{6}+15 n^{4}-7 n^{2}}{6}$ \\
\hline Continuous Lyapunov & $\frac{16 n^{6}+15 n^{4}-n^{2}}{6}$ \\
\hline Discrete Lyapunov & Proposed solutions - Use of vech \\
\hline \multicolumn{2}{|c|}{ Calculation Burden } \\
\hline Matrix Equation & $\frac{44 n^{6}+72 n^{5}+51 n^{4}+2 n^{3}-11 n^{2}-14 n}{24}$ \\
\hline Continuous Lyapunov & $\frac{44 n^{6}+72 n^{5}+51 n^{4}+2 n^{3}+13 n^{2}-14 n}{24}$ \\
\hline Discrete Lyapunov & $\frac{24}{24}$ \\
\hline \multicolumn{2}{|c|}{ Proposed solutions - Use of veck } \\
\hline Matrix Equation & $\frac{44 n^{6}-72 n^{5}+57 n^{4}+146 n^{3}-5 n^{2}-2 n}{24}$ \\
\hline Continuous Lyapunov & $\frac{44 n^{6}-72 n^{5}+57 n^{4}+242 n^{3}-53 n^{2}+22 n}{24}$ \\
\hline Discrete Lyapunov & $\frac{42 n}{2}$ \\
\hline
\end{tabular}

From Table III, it is clear that the calculation burdens of all solutions depend on the matrices dimension $n$.

From Table III, it is clear that the classical solutions via vec operator is of the order of $\frac{16}{6} \mathrm{n}^{6}$, while the proposed solutions via vech and veck operators are of the order of $\frac{44}{24} \mathrm{n}^{6}$, and hence the proposed solutions are faster than the classical solutions.

The proposed solutions which use vech operator are faster than the classical solutions which use vec operator, for $n \geq 4$. The proposed solutions which use veck operator are faster than the classical solutions which use vec operator, for $n \geq 2$. The solutions via veck operator are faster than the solutions via vech operator.

The calculation burdens of the classical and the proposed solutions of the continuous Lyapunov equation are shown in Fig.1.

The calculation burdens of the classical and the proposed solutions of the discrete Lyapunov equation are shown in Fig.2.

It is clear that from Table III we are able to compute the speedup form the classical solution via vec operator to the proposed solutions via vech and 
veck operators, of the continuous as well as the discrete Lyapunov equations.

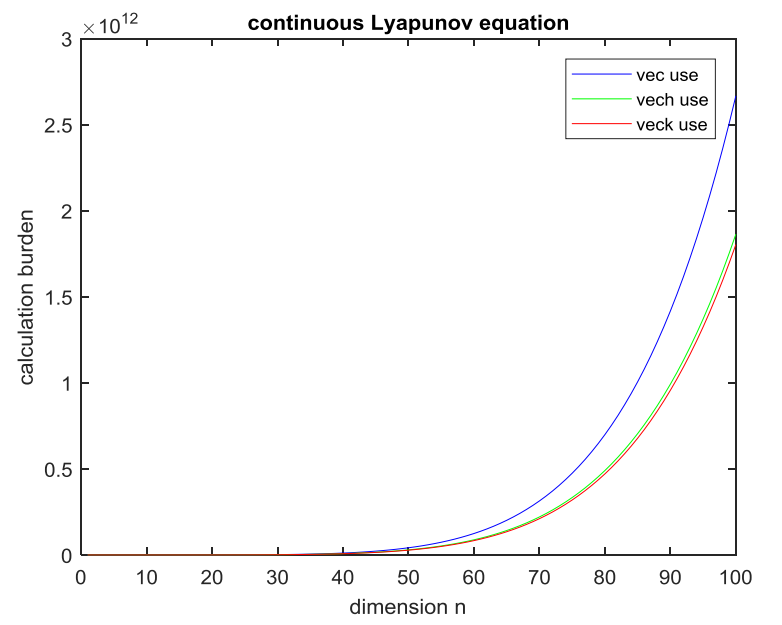

Fig. 1 Calculation burden of continuous Lyapunov equation: use of vec, vech and veck operators

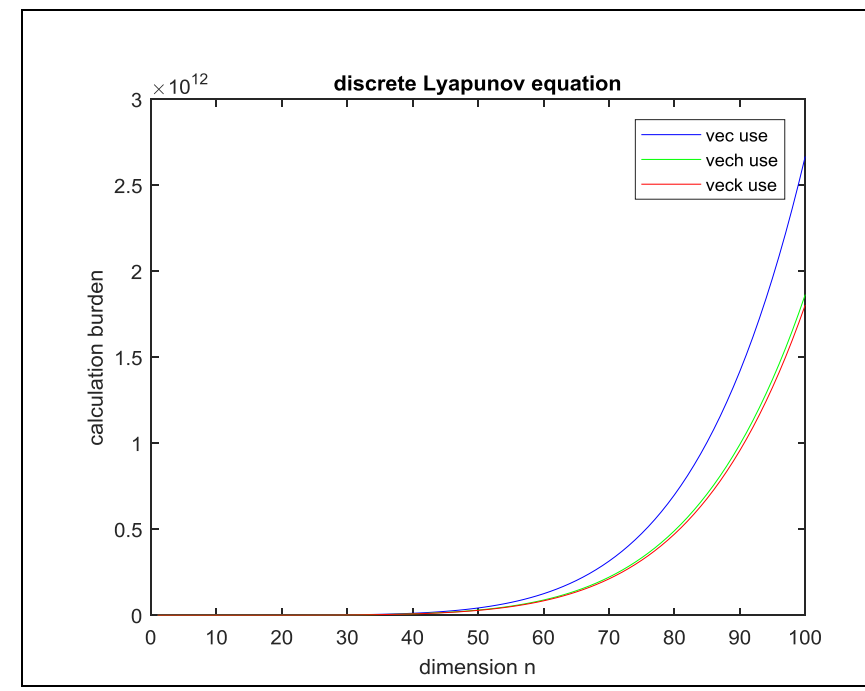

Fig. 2 Calculation burden of discrete Lyapunov equation: use of vec, vech and veck operators

The speedup form the classical to the proposed solution via vech operator of the continuous Lyapunov equation tends to the maximum speedup as $\mathrm{n}$ tends to infinity:

maxspeedup $=\frac{\frac{16}{6} \mathrm{n}^{6}}{\frac{44}{24} \mathrm{n}^{6}}=\frac{16}{11}=1.4545$

The speedup form the classical to the proposed solution via veck operator of the discrete Lyapunov equation tends to the minimum speedup as $\mathrm{n}$ tends to infinity:

minspeedup $=\frac{\frac{16}{6} \mathrm{n}^{6}}{\frac{44}{24} \mathrm{n}^{6}}=\frac{16}{11}=1.4545$

The speedup form the classical solution to the proposed solutions of the continuous Lyapunov equation are shown in Fig.3.
The speedup form the classical solution to the proposed solutions of the discrete Lyapunov equation are shown in Fig.4.

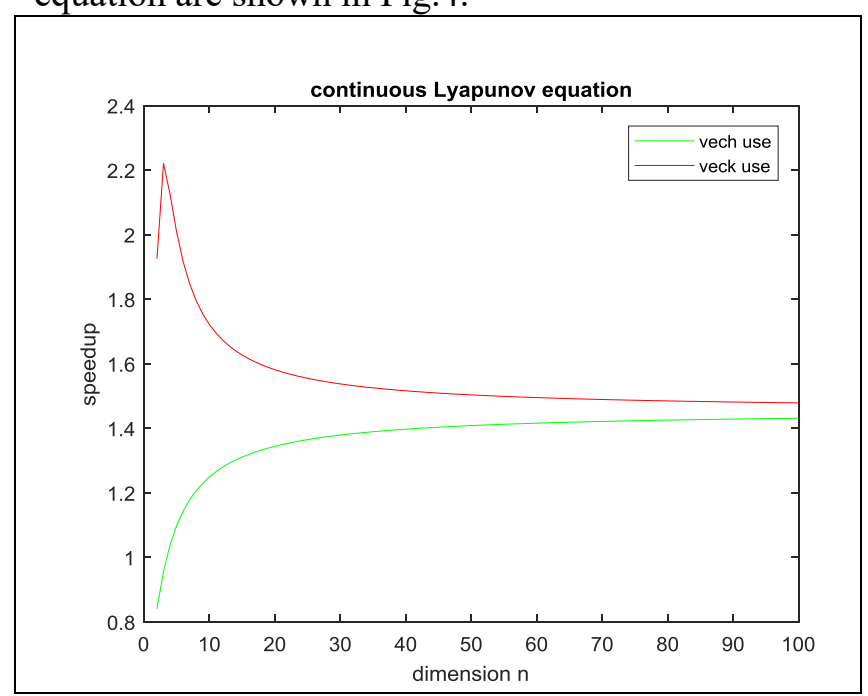

Fig. 3 Speedup of continuous Lyapunov equation solutions

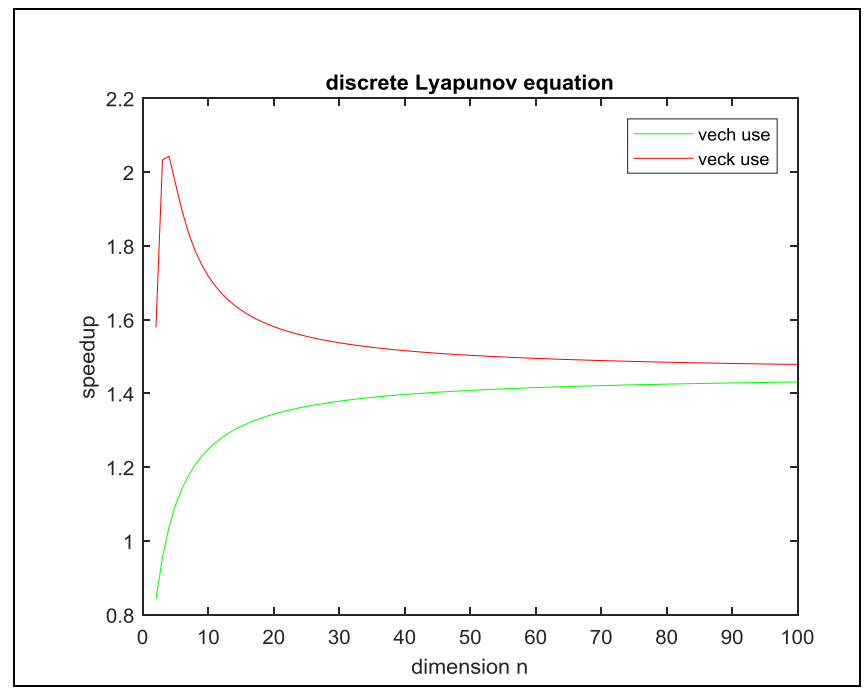

Fig. 4 Speedup of discrete Lyapunov equation solutions

\section{Conclusion}

Continuous and discrete Lyapunov equations are linear matrix equations, involving $n \times n$ dimensional matrices. The classical solution uses the vec operator. New closed forms are presented using the vech operator, due to the fact that Lyapunov equations involve symmetric matrices. New closed forms are presented using the veck operator, due to the fact that Lyapunov equations can be written in a form using skew symmetric matrices.

The classical solutions which use the vec operator require the inversion of a $\mathrm{n}^{2} \times \mathrm{n}^{2}$ dimensional matrix, while the proposed solutions which use the vech operator require the inversion of 
a $\frac{\mathrm{n}(\mathrm{n}+1)}{2} \times \frac{\mathrm{n}(\mathrm{n}+1)}{2}$ dimensional matrix and the proposed solutions which use the veck operator require the inversion of a $\frac{n(n-1)}{2} \times \frac{n(n-1)}{2}$ dimensional matrix.

The use of vech and veck instead of vec decreases the calculation burden. The proposed solutions are faster than the classical solutions. The proposed solutions which use vech operator are faster than the classical solutions which use vec operator, for $n \geq 4$. The proposed solutions which use veck operator are faster than the classical solutions which use vec operator, for $n \geq 2$. The solutions via veck operator are faster than the solutions via vech operator.

The maximum speedup form the classical to the proposed solution via vech operator of the continuous Lyapunov equation is 1.4545. The minimum speedup form the classical to the proposed solution via veck operator of the discrete Lyapunov equation is 1.4545 .

The main contribution of this paper concerns a) the analytic determination of the computational requirements of the classical algorithm via vec operator and the proposed algorithms via vech and veck operators, with respect to the matrices dimension $\mathrm{n}, \mathrm{b}$ ) the derivation of the speedup form the classical solution via vec operator to the proposed solutions via vech and veck operators.

\section{Appendix}

The calculation burdens of the classical and the proposed solutions for the general multidimensional case, where $n \geq 2$, are analytically calculated in Tables IV and V. Recall that $\mathrm{N}=\mathrm{n}^{2}, \mathrm{~m}=\frac{\mathrm{n}(\mathrm{n}+1)}{2}$ and $\mathrm{k}=\frac{\mathrm{n}(\mathrm{n}-1)}{2}$.

TABLE IV. CALCULATION BURDENS OF CONTINUOUS LyapunOV EQUATIONS SOLUTIONS

\begin{tabular}{|c|c|}
\hline \multicolumn{2}{|c|}{ Classical solution-Use of vec } \\
\hline Matrix Operation & Calculation Burden \\
\hline$I_{n} \otimes F$ & 0 \\
\hline$F \otimes I_{n}$ & 0 \\
\hline$C=I_{n} \otimes F+F \otimes I_{n}$ & $N^{2}$ \\
\hline$C^{-1}$ & $\frac{1}{6}\left(16 N^{3}-3 N^{2}-N\right)$ \\
\hline $\operatorname{vec}(X)=C^{-1} \cdot \operatorname{vec}(Q)$ & $2 N^{2}-N$ \\
\hline \multicolumn{2}{|c|}{ Proposed solution-Use of vech } \\
\hline Matrix Operation & Calculation Burden \\
\hline$I_{n} \otimes F$ & 0 \\
\hline$F \otimes I_{n}$ & 0 \\
\hline$C=I_{n} \otimes F+F \otimes I_{n}$ & $N^{2}$ \\
\hline$L_{n} \cdot C$ & $2 N^{2} m-N m$ \\
\hline
\end{tabular}

\begin{tabular}{|c|c|}
\hline \multicolumn{2}{|c|}{ Classical solution-Use of vec } \\
\hline Matrix Operation & Calculation Burden \\
\hline$E=L_{n} \cdot C \cdot D_{n}$ & $2 N m^{2}-m^{2}$ \\
\hline$E^{-1}$ & $\frac{1}{6}\left(16 m^{3}-3 m^{2}-m\right)$ \\
\hline $\operatorname{vech}(X)=E^{-1} \cdot \operatorname{vech}(Q)$ & $2 m^{2}-m$ \\
\hline \multicolumn{2}{|c|}{ Proposed solution-Use of veck } \\
\hline Matrix Operation & Calculation Burden \\
\hline$F \cdot Q$ & $2 n^{3}-n^{2}$ \\
\hline$R=F \cdot Q-Q \cdot F^{T}=F \cdot Q-(F \cdot Q)^{T}$ & $k$ \\
\hline$I_{n} \otimes F$ & 0 \\
\hline$F \otimes I_{n}$ & 0 \\
\hline$C=I_{n} \otimes F+F \otimes I_{n}$ & $N^{2}$ \\
\hline$C \cdot d_{n}$ & $2 N^{2} k-N k$ \\
\hline$d_{n}^{T} \cdot C \cdot d_{n}$ & $2 N k^{2}-k^{2}$ \\
\hline$D=\frac{1}{2} \cdot d_{n}^{T} \cdot C \cdot d_{n}$ & $k^{2}$ \\
\hline$D^{-1}$ & $\frac{1}{6}\left(16 k^{3}-3 k^{2}-k\right)$ \\
\hline $\operatorname{veck}(S)=D^{-1} \cdot \operatorname{veck}(-R)$ & $2 k^{2}-k$ \\
\hline$S-Q$ & $N$ \\
\hline$\frac{1}{2} \cdot(S-Q)$ & $N$ \\
\hline$F^{-1}$ & $\frac{1}{6}\left(16 n^{3}-3 n^{2}-n\right)$ \\
\hline$X=\frac{1}{2} \cdot F^{-1} \cdot(S-Q)$ & $2 n^{3}-n^{2}$ \\
\hline
\end{tabular}

TABLE V. CALCULATION BuRdENS OF Discrete LyaPunOV EQUATION SOLUTIONS

\begin{tabular}{|c|c|}
\hline \multicolumn{2}{|c|}{ Classical solution-Use of vec } \\
\hline Matrix Operation & Calculation Burden \\
\hline$I_{n} \otimes I_{n}$ & 0 \\
\hline$F \otimes F$ & $N^{2}$ \\
\hline$C=I_{n} \otimes I_{n}-F \otimes F$ & $N$ \\
\hline$C^{-1}$ & $\frac{1}{6}\left(16 N^{3}-3 N^{2}-N\right)$ \\
\hline $\operatorname{vec}(X)=C^{-1} \cdot \operatorname{vec}(Q)$ & $2 N^{2}-N$ \\
\hline \multicolumn{2}{|c|}{ Proposed solution - Use of vech } \\
\hline Matrix Operation & Calculation Burden \\
\hline$I_{n} \otimes I_{n}$ & 0 \\
\hline$F \otimes F$ & $N^{2}$ \\
\hline$C=I_{n} \otimes I_{n}-F \otimes F$ & $N$ \\
\hline$L_{n} \cdot C$ & $2 N^{2} m-N m$ \\
\hline$E=L_{n} \cdot C \cdot D_{n}$ & $2 N m^{2}-m^{2}$ \\
\hline$E^{-1}$ & $\frac{1}{6}\left(16 m^{3}-3 m^{2}-m\right)$ \\
\hline $\operatorname{vech}(X)=E^{-1} \cdot \operatorname{vech}(Q)$ & $2 m^{2}-m$ \\
\hline \multicolumn{2}{|c|}{ Proposed solution-Use of veck } \\
\hline Matrix Operation & Calculation Burden \\
\hline$F \cdot Q$ & $2 n^{3}-n^{2}$ \\
\hline$R=F \cdot Q-Q \cdot F^{T}=F \cdot Q-(F \cdot Q)^{T}$ & $k$ \\
\hline$I_{n} \otimes I_{n}$ & 0 \\
\hline$F \otimes \mathrm{F}$ & $N^{2}$ \\
\hline$C=I_{n} \otimes I_{n}-F \otimes \mathrm{F}$ & $N$ \\
\hline$C \cdot d_{n}$ & $2 N^{2} k-N k$ \\
\hline$d_{n}^{T} \cdot C \cdot d_{n}$ & $2 N k^{2}-k^{2}$ \\
\hline$D=\frac{1}{2} \cdot d_{n}^{T} \cdot C \cdot d_{n}$ & $k^{2}$ \\
\hline$D^{-1}$ & $\frac{1}{6}\left(16 k^{3}-3 k^{2}-k\right)$ \\
\hline
\end{tabular}




\begin{tabular}{|c|c|}
\hline $\operatorname{veck}(S)=D^{-1} \cdot \operatorname{veck}(R)$ & $2 k^{2}-k$ \\
\hline$F \cdot S$ & $2 n^{3}-n^{2}$ \\
\hline$Q-F \cdot S$ & $n^{2}$ \\
\hline$F \cdot F$ & $2 n^{3}-n^{2}$ \\
\hline$I-F \cdot F$ & $n$ \\
\hline$(I-F \cdot F)^{-1}$ & $\frac{1}{6}\left(16 n^{3}-3 n^{2}-n\right)$ \\
\hline$X=(I-F \cdot F)^{-1} \cdot(Q-F \cdot S)$ & $2 n^{3}-n^{2}$ \\
\hline
\end{tabular}

References:

[1] P. Lancaster P., M. Tismenetsky, The Theory of Matrices. Academic Press, Orlando, 2nd edition, 1985.

[2] R.A. Horn, C.R. Johnson, Topics in Matrix Analysis. Cambridge University Press, Cambridge, 1991.

[3] P. Benner, Control Theory Basics, Chapter 57 of the Handbook of Linear Algebra, Leslie Hogben, Chapman \& Hall/CRC, 2006.

[4] A. Nakhmani., Modern Control: State-Space Analysis and Design Methods, McGraw Hill, 2020.

[5] B.D.O. Anderson and J.B. Moore, Optimal Filtering, Dover Publications, New York, 2005.

[6] M. A. Hamadi, K. Jbilou and A. Ratnani, A model reduction method in large scale dynamical systems using an extended-rational block Arnoldi method, Journal of Applied Mathematics and Computing, 2021, https://doi.org/10.1007/s12190-021-01521-0

[7] V. Simoncini, A New Iterative Method for Solving Large-Scale Lyapunov Matrix Equations, SIAM Journal on Scientific Computing, vol. 29, no. 3, 2007, pp. 12681288.

[8] E.L. Wachspress, Iterative Solution of the Lyapunov Matrix Equation, Appl. Mulh. Left., vol. I, no. I, 1988, pp. 87-90.

[9] D. Rothschild \& A. Jameson, Comparison of four numerical algorithms for solving the Liapunov matrix equation, International Journal of Control, vol. 11, no. 2, 1970, pp. 181-198.

[10] D.S. Bernstein, Matrix Mathematics, Theory, Facts, and Formulas, Princeton University Press, Princeton, NJ, USA, 2nd edition, 2009.

[11] D.A. Harville, Matrix Algebra from a statistician's perspective, Springer-Verlag New York, 1997.

[12] E.W. Grafarend, Linear and nonlinear models: fixed effects, random effects, and mixed models, De Gruyter, 2006.

[13] H. Sato, Riemannian Newton's method for joint diagonalization on the Stiefel manifold with application to ICA, arXiv:1403.8064v2 [math.OC], 2014.

[14] Z. Gajic, M.T.J. Qureshi, Lyapunov Matrix Equation in System Stability and Control, Dover Publications Inc., Mineola, New York, 1995.

[15] B. Datta, Numerical solutions and conditioning of Lyapunov and Sylvester equations, in Numerical Methods for Linear Control Systems, Elsevier Academic Press, 2004.

[16] N. Assimakis and M. Adam, Discrete time Kalman and Lainiotis filters comparison, Int. Journal of Mathematical Analysis (IJMA), vol. 1, no. 13, 2007, pp. 635-659.

\section{Contribution of individual authors to the creation of a scientific article (ghostwriting policy)}

Nicholas Assimakis: Conceptualization, Methodology, Software, Validation.

Maria Adam: Formal analysis, Investigation, Writing - original draft preparation, Writing review and editing and Visualization.

\section{Creative Commons Attribution \\ License 4.0 (Attribution 4.0 \\ International , CC BY 4.0)}

This article is published under the terms of the Creative Commons Attribution License 4.0 